\title{
Atualizações no Tratamento do Lúpus Cutâneo
}

\section{Updates in Cutaneous Lupus Treatment}

\author{
Luiza Helena Ribeiro ${ }^{(1)}$, Maria José Nunes ${ }^{(2)}$, Andréa Barranjard Vannucci Lomonte ${ }^{(2)}$, Luis Carlos Latorre(3)
}

\section{RESUMO}

O lúpus cutâneo é a principal manifestação clínica do lúpus eritematoso sistêmico. Diferentes formas de apresentações clínicas e histopatológicas compõem o quadro cutâneo. A diferenciação de tais apresentações clínicas é de grande relevância para melhor abordagem terapêutica dos pacientes. Ampla variedade de intervenções terapêuticas está atualmente disponível para o tratamento do lúpus cutâneo. Nessa revisão, serão abordadas as principais opções terapêuticas, medicamentosas e não-medicamentosas, além das novas terapias ainda em estudo.

Palavras-chave: lúpus eritematoso sistêmico, lúpus cutâneo, classificação, tratamento.

\section{INTRODUÇÃO}

O termo lúpus (lobo em latim) vem sendo utilizado desde o período medieval para descrever diferentes tipos de lesões cutâneas com aparência da face de um lobo. Entretanto, quem primeiro descreveu as manifestações clínicas do lúpus sistêmico e cutâneo foi Cazenave ${ }^{(1)}$.

Desde então, diferentes definições e termos discordantes vêm sendo utilizados para denominar as manifestações cutâneas da doença ${ }^{(2)}$. Até mesmo dados epidemiológicos que envolvem a prevalência de tais manifestações são imprecisos, principalmente pelo fato de serem relatados por dois especialistas distintos: reumatologistas e dermatologistas.

Apesar disso, o envolvimento cutâneo do lúpus eritematoso é uma das principais manifestações clínicas da doença. Sua diferenciação, tanto em relação às características clínicas quanto histológicas, é de grande relevância para a abordagem terapêutica ${ }^{(3)}$.

\section{CLASSIFICAÇÃO}

As manifestações cutâneas específicas do lúpus dividemse em duas classificações: uma clínica e outra histológica.

\begin{abstract}
Cutaneous lupus is the most common systemic lupus erythematosus clinical manifestation. Skin lesions are classified according to the clinical and histopathologic features, which are of great relevancy for the management of the disease. Nowadays several therapeutic interventions are available to the treatment of cutaneous lupus. In this review we will approach the most widely used and also the new therapies which are under study.
\end{abstract}

Keywords: lupus eritematosus cutaneous, systemic lupus erythematosus, classification, treatment, managment.

São três as formas de apresentação clínica do lúpus eritematoso: agudo, subagudo e crônico. As apresentações histológicas distribuem-se nas formas agudas, subagudas (variantes anular e psoriasiforme) e crônicas (lúpus discóide, tumidus e profundus) (Figuras 1, 2 e 3).

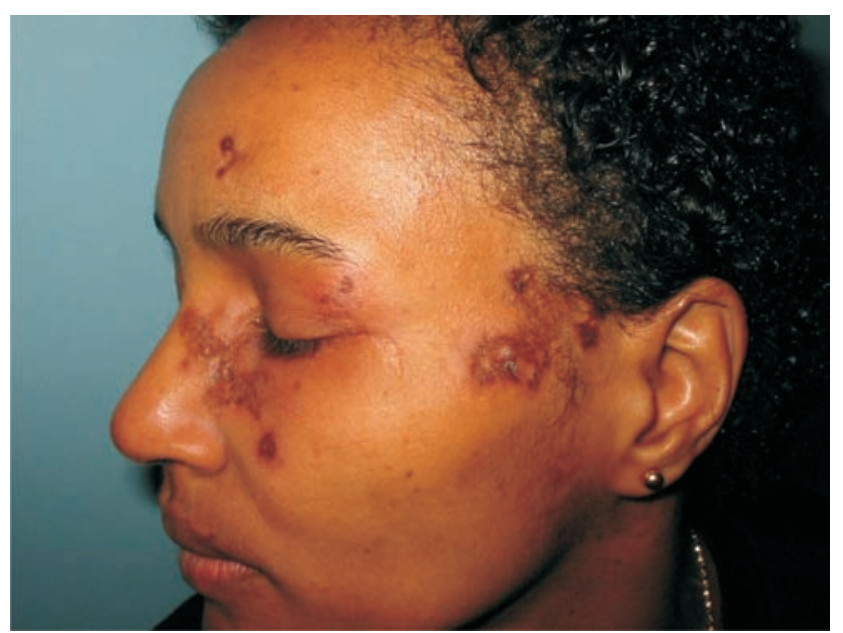

Figura 1 - Lúpus discóide: placas hiperpigmentadas e lesões cicatriciais.

Recebido em 8/1/2008. Aprovado, após revisão, em 19/8/2008. Declaramos a inexistência de conflitos de interesse Serviço de Reumatologia do Hospital Heliópolis.

1. Médica reumatologista, mestre em Reumatologia pela Universidade Federal de São Paulo (Unifesp)

2. Médica reumatologista.

3. Médico reumatologista, doutor em Saúde Pública pela Universidade de São Paulo (USP).

Endereço para correspondência: Luiza Helena Ribeiro, Av. Almirante Delamare, 1534, Setor Rosa, 04230-000, São Paulo, SP, e-mail: luizahcr@hotmail.com 


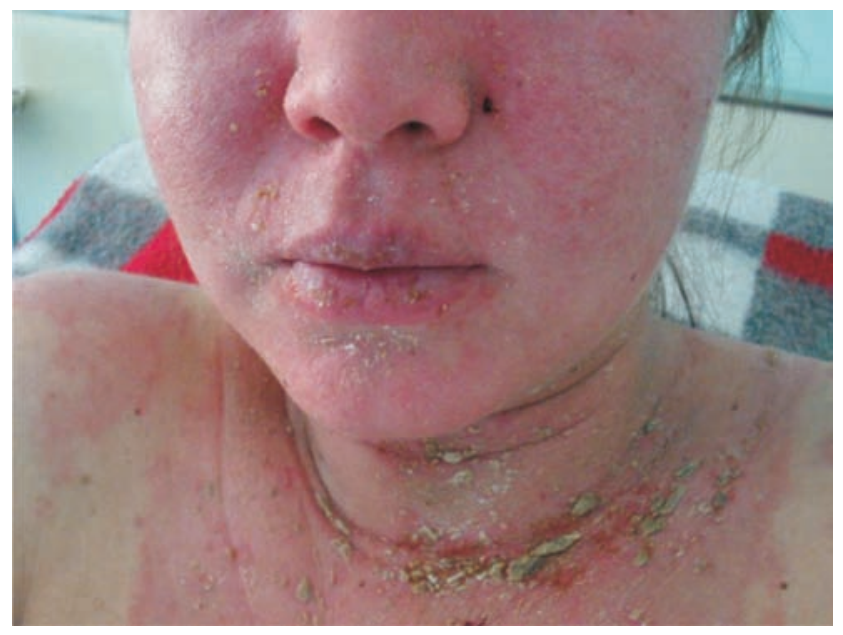

Figura 2 - Lúpus subagudo: predomínio de lesões psoriasiformes.

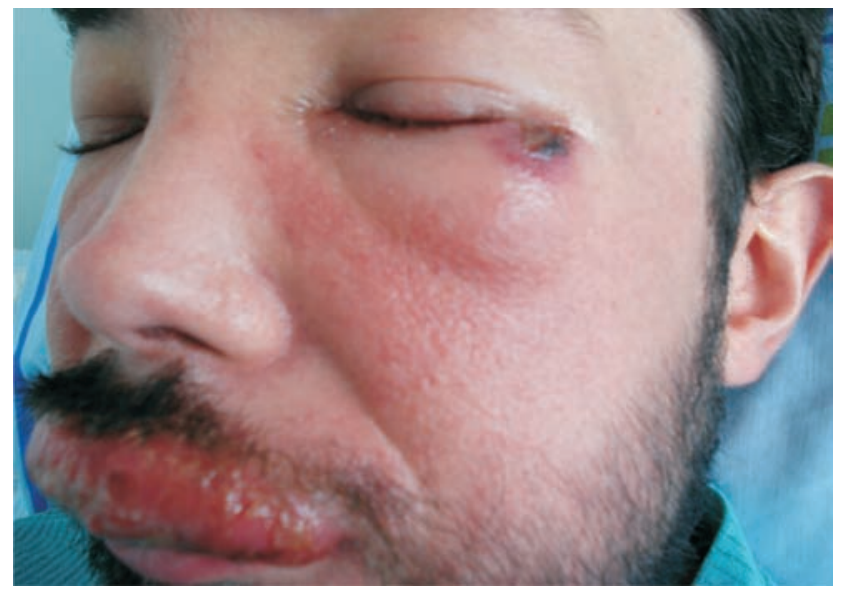

Figura 3 - Lúpus tumidus: lesões infiltrativas com intenso edema.

Entretanto, essa distribuição classificatória tem finalidade meramente didática, uma vez que formas distintas podem acometer o mesmo paciente ${ }^{(2,4)}$ (Tabela 1$)$.

Tabela 1

ClassificaÇÃo do LÚPUS ERITEMATOSO CUTÂNeO

\begin{tabular}{|c|}
\hline $\begin{array}{l}\text { Lúpus eritematoso agudo } \\
\text { Localizado } \\
\text { Generalizado }\end{array}$ \\
\hline $\begin{array}{l}\text { Lúpus eritematoso subagud } \\
\text { Anular } \\
\text { Psoriasiforme }\end{array}$ \\
\hline $\begin{array}{l}\text { Lúpus eritematoso crônico } \\
\text { Discóide } \\
\text { Tumidus } \\
\text { Profundus }\end{array}$ \\
\hline
\end{tabular}

\section{LÚPUS ERITEMATOSO CUTÂNEO AGUDO}

As lesões cutâneas surgem durante a atividade sistêmica da doença. Uma associação de lesões é vista, sendo as mais comuns o rash malar, o eritema maculopapular, a fotossensibilidade, a alopecia, as aftas orais e as lesões vasculíticas. As lesões do lúpus agudo apresentam-se clinicamente nas formas localizadas (eritema malar) e generalizadas (lesões disseminadas pelo corpo). Os achados imunopatológicos típicos das lesões agudas são de dermatite, sem significativa hiperceratose ou atrofia da epiderme $e^{(1,5)}$.

\section{LÚPUS ERITEMATOSO CUTÂNEO SUBAGUDO}

Clinicamente as lesões são apresentadas como placas não infiltrativas que preferencialmente envolvem as áreas expostas do tronco e das extremidades superiores. São divididas em lesões do tipo anular e psoriasiforme. As lesões anulares têm menor acometimento da epiderme e têm distribuição mais centrípeta que centrífuga, como no caso das lesões psoriasiformes, que também se caracterizam pela significativa descamação.

As lesões subagudas correlacionam-se com a presença de anticorpos anti-Ro.

Exames histológicos revelam o envolvimento da derme e da epiderme. A variante anular não tem envolvimento de anexos, ao contrário das psoriasiformes ${ }^{(1)}$.

\section{LÚPUS CUTÂNEO CRÔNICO}

As lesões crônicas clássicas são as lesões discóides. Caracterizam-se como placas recobertas por fino tecido descamativo, que se estende até o folículo piloso. As placas podem ser inicialmente hiperpigmentadas e em um processo evolutivo se despigmentarem e evoluírem para lesões cicatriciais mais profundas, que são, na maioria das vezes, permanentes. A histologia mostra infiltrado predominantemente linfocítico na junção derme-epiderme. Ao contrário das lesões agudas subagudas, o envolvimento de anexos e a atrofia da derme são achados freqüentes ${ }^{(6)}$.

O lúpus tumidus é caracterizado por lesões tipo pápulas urticariformes, nódulos ou placas infiltrativas edemaciadas que atingem preferencialmente a cabeça, o pescoço e os $\operatorname{braços}^{(7)}$. Achados histológicos revelam infiltrado linfocítico perivascular e perianexial e presença característica de mucina ${ }^{(8)}$.

O lúpus profundus é também denominado paniculite lúpica. Suas lesões são mais profundas e caracterizadas pela presença de nódulos e placas. Acometem superfícies expostas a traumas, como bochechas, ombros, coxas e mamas. A progressão das lesões pode resultar calcificação e ulcerações. 
A presença de paniculite linfocítica é o achado histológico predominante $^{(9)}$.

\section{TRATAMENTO}

\section{MEDIDAS GERAIS}

Hábitos de vida têm forte correlação com o surgimento e o agravamento das lesões cutâneas do lúpus ${ }^{(10)}$.

A exposição solar é um dos fatores externos envolvidos na patogênese da doença, se não o principal. Vários estudos, ao longo dos anos, têm discutido o papel da radiação ultravioleta nos eventos imunológicos envolvidos na patogênese do lúpus eritematoso sistêmico. Mais recentemente, a indução da apoptose de queratinócitos, por meio da exposição à radiação UVA $\mathrm{e}$, particularmente UVB, tem sido avaliada ${ }^{(11-14)}$.

Os pacientes devem ser orientados quanto aos riscos da exposição ao sol e da necessidade de uso de guarda-sol, sombrinhas, bonés etc., além de protetor solar.

Os protetores solares têm papel fundamental no arsenal terapêutico do lúpus cutâneo. São agentes químicos que absorvem a luz ultravioleta (UV), podendo bloquear as radiações UVA, UVB ou ambas. Diferentes veículos podem ser utilizados, como creme, óleo, gel, álcool ou loção. Embora se possa atingir maior proteção solar com os maiores níveis de fator protetor (variam de 2 a 50 ), a diferença entre o bloqueio solar do fator 15 (93\% de proteção) e do fator 50 é de apenas $5 \%(98 \% \text { de proteção })^{(10)}$.

Outro hábito de vida que tem sido relacionado ao lúpus é o de fumar. $\mathrm{O}$ tabagismo tem sido implicado na patogênese do lúpus eritematoso sistêmico, na formação de auto-anticorpos e em maior gravidade das lesões ${ }^{(15-18)}$. Estudos relatam menor eficácia da cloroquina em indivíduos fumantes por causa do efeito do tabaco no citocromo P450, cujo sistema enzimático é responsável pelo metabolismo da cloroquina ${ }^{(19,20)}$.

Estudos controlados não demonstraram que hábitos alimentares estariam diretamente relacionados à atividade do lúpus eritematoso ${ }^{(10)}$. No entanto, as complicações da doença exigem alimentação mais saudável, com restrições dietéticas.

\section{CORTICOSTERÓIDES}

CORTICÓIDES TÓPICOS

O tratamento local é usado para lesões isoladas ou refratárias e atualmente tem-se diversas preparações disponíveis. Os corticosteróides (CE) podem ser divididos em fluorados e não-fluorados e podem ser de baixa, média e alta potências (Tabela 2). A maioria dos CE não-fluorados inclui a hidrocortisona. Apesar de serem mais baratos, eles são menos potentes do que os fluorados, os quais produzem mais efeitos colaterais, como atrofias, despigmentações, estrias, telangectasias, acne, foliculites e superinfecção por Cândida ${ }^{(21)}$; conseqüentemente, não devem ser usados por mais de duas semanas. A betametasona e o clobetasol, particularmente, são bastante efetivos especialmente em associação com antimaláricos ${ }^{(22)}$. Pomadas geralmente são mais efetivas que cremes, géis ou loções. Há, também, preparações na forma de patches que permitem melhor absorção dos CE de alta potência com menos irritação.

As lesões mais crônicas respondem pobremente à terapia com CE tópico.

Tabela 2

CORTICOSTERÓIDES TÓPICOS: POTÊNCIA E FORMULAÇÃO

\begin{tabular}{|c|c|c|}
\hline Potência & Medicação & Formulação \\
\hline Hidrocortisona & Baixa & $\begin{array}{l}\text { Creme, pomada ou loção } \\
\text { a } 2,5 \% \text { ou } 1,0 \%\end{array}$ \\
\hline Valerato de betametasona & Média & Creme a $0,1 \%$ \\
\hline Valerato de hidrocortisona & & Creme ou pomada a $0,2 \%$ \\
\hline Acetonido de triancinolona & & $\begin{array}{c}\text { Creme, pomada ou loção } \\
\text { a } 0,1 \% \text { ou } 0,025 \%\end{array}$ \\
\hline Dipropionato de betametasona & Alta & Creme ou pomada a $0,05 \%$ \\
\hline Valerato de betametasona & & Pomada a $0,1 \%$ \\
\hline Acetonida de fluocinolona & & Creme a $0,2 \%$ \\
\hline Halcinonida & & Creme ou pomada a $0,1 \%$ \\
\hline Propionato de clobetasol & Muito Alta & Creme ou pomada a $0,05 \%$ \\
\hline Propionato de halobetasol & & Creme ou pomada a $0,05 \%$ \\
\hline
\end{tabular}

\section{CORTICÓIDES SISTÊMICOS}

Pacientes com doença sistêmica grave associada ao quadro cutâneo necessitam de tratamento com CE, podendo ser utilizada a prednisona oral ou a pulsoterapia com metilprednisolona. Entretanto, na ausência de doença sistêmica e por causa de seus efeitos colaterais potenciais, é preferível o uso de antimaláricos, retinóides ou imunossupressores nos casos de lesões cutâneas eritematosas ou discóides mais persistentes, que requerem altas doses de $\mathrm{CE}$, ou lesões que recorram ao se diminuir a dose destes ${ }^{(23)}$.

\section{ANTIMALÁRICOS}

Os primeiros estudos clínicos demonstrando a eficácia do tratamento do lúpus eritematoso foram descritos no 
tratamento do lúpus discóide, utilizando empiricamente a quinina e a quinacrina ${ }^{(24)}$. Desde então, não obstante virem sendo utilizados para o tratamento de outras manifestações clínicas do lúpus eritematoso sistêmico, seu papel proeminente é na melhor resposta terapêutica das manifestações cutâneas, especialmente no lúpus discóide ${ }^{(25)}$.

Nos pacientes resistentes à monoterapia, foi previamente relatada em alguns estudos ${ }^{(26,27)}$ boa resposta à terapia com combinação de antimaláricos.

Os antimaláricos mais estudados e utilizados são a hidroxicloroquina, a quinacrina e o difosfato de cloroquina. A dose recomendada da hidroxicloroquina é de $6,5 \mathrm{~m} / \mathrm{kg} /$ dia. A maior precaução com o seu uso deve ser quanto a sua toxidade retiniana ${ }^{(4)}$.

Como foi anteriormente descrito, a cloroquina parece ser menos eficaz em indivíduos fumantes ${ }^{(17-20)}$.

\section{RETINÓIDES}

Os retinóides sintéticos isotretinoína e acitretina são conhecidos como drogas de segunda linha para tratamento sistêmico do lúpus eritematoso cutâneo (LEC), sendo opção quando há falha no uso de antimaláricos. Os retinóides têm sido empregados com sucesso em casos de LEC subagudo e crônico, em doses que variam de 0,5 a $1 \mathrm{mg} / \mathrm{kg} /$ dia, sendo particularmente úteis em casos de lúpus discóide ${ }^{(28)}$.

Um estudo aberto relatou tratamento com isotretinoína em pacientes com LEC subagudo e crônico. Houve melhora clínica e histológica das lesões de pele, porquanto as melhores respostas foram vistas em pacientes com LEC crônico. A tolerância à droga foi boa ${ }^{(29)}$.

Outro estudo de curta duração, randomizado, envolvendo 58 pacientes com lúpus eritematoso discóide, comparou o uso de acitretina com hidroxicloroquina. Melhora clínica foi observada em cerca de $50 \%$ dos pacientes de ambos os grupos, mas os efeitos adversos foram mais freqüentes e mais graves no grupo acitretina ${ }^{(30)}$.

$\mathrm{O}$ uso a longo prazo de retinóides é freqüentemente limitado pelos efeitos colaterais potenciais, que requerem monitorização e incluem: hepatite medicamentosa, hipertrigliceridemia, secura cutânea e de mucosas, alterações ósseas consistentes com hiperostose esquelética idiopática difusa (DISH) e teratogenicidade, sendo obrigatório o uso de métodos contraceptivos. Além disso, é aconselhável o uso cuidadoso de protetores solares, já que os retinóides podem agravar a fotossensibilidade ${ }^{(31)}$.

\section{IMUNOSSUPRESSORES}

Não há evidência suficiente na literatura sobre o emprego de imunossupressores no tratamento do $\operatorname{LEC}^{(32,33)}$. Todavia, lesões graves que parecem ser irreversíveis podem, de fato, apresentar melhora com o tratamento imunossupressor mais agressivo, que muitas vezes é iniciado para manifestações mais graves do lúpus, como nefrite ou cerebrite. Sendo assim, essas drogas constituem terapia de terceira linha no tratamento do LEC.

\section{AZATIOPRINA}

A azatioprina pode ser útil em várias formas de LEC, incluindo o lúpus eritematoso discóide.

Há um relato de caso na literatura em que a azatioprina foi empregada no tratamento de seis pacientes com LEC (quatro com LEC subagudo e dois com LEC discóide) e em seis pacientes com vasculite leucocitoclástica cutânea crônica. Todos os pacientes eram refratários ao tratamento convencional e necessitaram do uso de corticosteróide oral por tempo prolongado para controle da doença. Entre os pacientes com LEC, três apresentaram boa resposta, com melhora quase completa das lesões de pele, permitindo a redução da dose da prednisona. Um dos pacientes teve melhora inicial, mas desenvolveu pancreatite. $\mathrm{O}$ tratamento falhou em dois pacientes (um teve náusea e o outro teve febre). Dos pacientes com vasculite leucocitoclástica, cinco apresentaram melhora ${ }^{(34)}$.

Reações adversas associadas ao uso da azatioprina incluem: intolerância gastrintestinal, toxicidade da medula óssea, aumento da suscetibilidade a infecções, hepatite aguda e pancreatite ${ }^{(35)}$.

\section{Metotrexato}

A experiência clínica com metotrexato (MTX) no lúpus eritematoso sistêmico tem sido limitada a relatos de casos e estudos abertos com doses similares a usadas para artrite reumatóide ${ }^{(36)}$. Os estudos clínicos têm enfatizado benefícios nas manifestações predominantemente sistêmicas (articular, constitucionais e serosas) e também cutâneas, como alternativa aos antimaláricos e baixas doses de corticóide ${ }^{(37-39)}$.

\section{LEFLUNOMIDA}

A leflunomida é um derivado isoxazólico, aprovado para o tratamento da artrite reumatóide, que inibe a síntese das pirimidinas, portanto, com propriedades antiinflamatórias. A droga apresenta extensiva recirculação enteroepática e é fortemente ligada a proteínas plasmáticas. Alguns estudos não controlados sugerem eficácia com doses padronizadas 
para artrite reumatóide, mas grandes ensaios com placebo são necessários para melhor compreensão do papel deste medicamento no lúpus eritematoso sistêmico, particularmente, nas manifestações cutâneas ${ }^{(40)}$.

Relatos de caso ou de séries de casos têm demonstrado que pacientes usuários do leflunomida podem induzir ou exacerbar o lúpus cutâneo subagudo. A causa de tal fenômeno é ainda desconhecida, embora seja sugerido que a inibição do fator de necrose tumoral, provocada pela leflunomida, esteja envolvida no mecanismo etiopatogênico ${ }^{(41-44)}$.

A dapsona (diaminodifenilsulfona) é ocasionalmente usada, tendo a sua melhor indicação nas lesões vesículobolhosas ${ }^{(45)}$. Alguns estudos envolvendo pacientes com lesões cutâneas refratárias à cloroquina obtiveram resultados favoráveis ao uso da dapsona ${ }^{(46)}$. Uma revisão avaliou a efetividade da dapsona no lúpus profundo ${ }^{(47)}$. Esse medicamento requer monitorização freqüente em razão de sua toxicidade hematológica, renal e hepática.

\section{TALIDOMIDA}

Embora seu uso tenha sido bastante limitado em virtude dos catastróficos efeitos teratogênicos da talidomida, além da neuropatia sensorial, essa droga mostra-se efetiva no tratamento das manifestações cutâneas refratárias do lúpus subagudo e do lúpus discóide ${ }^{(28,48,49)}$. A dose utilizada pode variar entre 50 a $100 \mathrm{mg} / \mathrm{dia}^{(31)}$.

\section{Micofenolato dE MOFETILA}

Não há estudos controlados por placebo que dêem suporte ao uso do micofenolato de mofetila (MMF) como droga de primeira linha para o tratamento do LEC.

Alguns relatos de caso mostraram bons resultados com o uso de micofenolato no tratamento das lesões cutâneas do lúpus não-responsivas ao tratamento com antimaláricos e outros agentes imunossupressores ${ }^{(50,51)}$.

Estudos mais recentes têm demonstrado resposta clínica satisfatória na regressão das lesões cutâneas com o uso do $\mathrm{MMF}^{(52,53)}$.

Os efeitos adversos do MMF incluem: intolerância gastrintestinal (principalmente diarréia), leucopenia e infecções. É recomendado o ajuste da dose em pacientes com insuficiência renal e seu uso é desaconselhado durante a gestação ${ }^{(35)}$.

\section{CicLofosfamida}

A ciclofosfamida é um agente alquilante considerado padrão-ouro para o manejo das formas graves do lúpus eritematoso sistêmico, particularmente na nefrite e nas desordens neurológicas, sendo de uso infreqüente nas manifestações predominantemente cutânea ${ }^{(52)}$.

\section{INIBIDORES DA CALCINEURINA}

O tacrolimo e o pimecrolimo são inibidores tópicos da calcineurina, cujos efeitos imunomoduladores impedem a ativação dos linfócitos $\mathrm{T}$ e a produção de citocinas ${ }^{(53)}$. Foram inicialmente desenvolvidos para o tratamento da dermatite atópica. Estudos mais recentes demonstraram seus efeitos benéficos em doenças cutâneas inflamatórias, como alternativa aos $\mathrm{CE}$ tópicos, por terem mecanismo de ação mais seletivo e por apresentarem menos efeitos adversos, como a atrofia cutânea e menor absorção sistêmica ${ }^{(54,55)}$.

A experiência com os inibidores da calcineurina em pacientes com lúpus cutâneo ainda é limitada, embora alguns estudos demonstrem bons resultados em pacientes com LEC subagudo e lúpus eritematoso sistêmico. Em casos de lúpus discóide não parecem ser efetivos, pela má penetração nas placas hipertróficas ${ }^{56,57)}$.

A aplicação deve ser feita duas vezes ao dia e os principais efeitos colaterais são: sensação de queimação, prurido e eritema, que geralmente melhoram com a continuidade do tratamento ${ }^{(58)}$.

\section{TERAPIA INTRALESIONAL}

Quando uma resposta satisfatória ao tratamento tópico das lesões cutâneas não é obtida, a terapia intralesional é uma alternativa. Alguns estudos demonstram resultados positivos do tratamento. A droga de escolha é a triancinolona em suspensão: diacetato de triancinolona a $1,25 \%$ ou $2,25 \%$ ou a triancinolona acetonida ${ }^{(10,59)}$.

A infiltração intralesional com quinacrina e cloroquina foi muito utilizada na década de 1950 , porém a alta incidência de efeitos adversos, como sangramentos, eritema e desconforto no local da aplicação, levou ao abandono de tal terapia ${ }^{(60,61)}$.

\section{TERAPIAS EXPERIMENTAIS E CIRURGIA}

Estudos preliminares têm demonstrado possíveis benefícios do uso de novas terapias no tratamento de lúpus cutâneo, refratário às terapias convencionais.

Novos medicamentos estão em investigação. Os agentes biológicos já aprovados para o tratamento de outras doenças reumáticas auto-imunes, como infliximabe, rituximabe, abatacepte, etarnecepte, além de novas terapias em investigação (anti-CD40, anti-C5, anti-BLyS e anti-IL10), têm mostrado resultados promissores em modelos animais e 
ensaios em humanos, particularmente nas manifestações sistêmicas graves da doençą ${ }^{(62-66)}$.

Estudos abertos utilizando aplicações locais de alfainterferon recombinante têm mostrado resultados promissores, porém com reaparecimento das lesões após término da terapia ${ }^{(67,68)}$. Além disso, alguns autores mostram-se apreensivos quanto ao risco de indução ou exacerbação de reações auto-imunes ${ }^{(2)}$. Uma possível relação do interferon tipo I na patogenia do lúpus e especialmente das suas manifestações cutâneas abrem espaço para o desenvolvimento de novas opções terapêuticas ${ }^{\left({ }^{69}\right)}$.

A fototerapia com radiação UVA-I em baixas doses (340 a $400 \mathrm{~nm}$ ) também tem sido estudada, embora exija grande precaução na avaliação dos resultados, por causa dos efeitos deletérios já longamente estudados das radiações ultravioletas no lúpus cutâneo ${ }^{(70-72)}$.

Outras terapias intralesionais experimentais são aplicação de sais de ouro, ácido cáustico, fluoracil tópico, nitrogênio mostarda, análogos da vitamina $\mathrm{D}$ e dióxido de carbono sólido ${ }^{(10)}$.

Tratamentos cirúrgicos estéticos têm limitado valor nas lesões crônicas cicatriciais, principalmente diante do risco de exacerbação da doença por causa do trauma cirúrgico ${ }^{(2)}$. Entretanto, estudos recentes utilizando procedimentos como

\section{REFERÊNCIAS}

1. Rowell NR: Some historical aspects of skin disease in lupus erythematosus. Lupus 6: 76-83, 1997.

2. Rothfield NF, Braverman IM, Moschella S, et al.: Classification of lupus erythematosus. An open forum. Fitzpatrick's. J Clin Dermatol 1: 9-12, 1994.

3. Sontheimer RD: Subacute cutaneous lupus erythematosus: a decade's perspective. Med Clin North Am 73:1073-90, 1989.

4. Mimouni D, Nousari CH: Lupus erytematosus and the skin. In: Lahita RG, editor: Systemic lupus erythematosus. 4. ed. Elsevier, Academic Press;. p. 855-76, 2004.

5. David-Bajar KM, Davis BM: Pathology, immunopathology and immunohistochemistry in cutaneous lupus erythematosus. Lupus 6: 145-57, 1997.

6. Wallace DJ: The relatioship between discoid and systemic lupus erythematosus. In: DJ Wallace, Hahn BH, Quismorio FP, Klinemberg JR, editors: Dubois' lupus erythematosus. Philadelphia and London: Lea \& Febiger; 1993. p. 310-2.

7. Kuhn A, Richter-Hintz D, Oslislo, et al.: Lupus erythematosus tumidus- a neglected subset of cutaneous lupus erythematosus : report of 40 cases. Arch Dermatol 136: 1033-41, 2000.

8. Dekle CL, Mannes KD, Davis LS, Sangueza OP: Lupus tumidus. J Am Acad Dermatol 41: 250-3, 1999.

9. Watanabe T, Tsuchida T: Lupus erythematosus a cutaneous marker for a distinct, profundus clinical subset? Br J Dermatol 134: 123-5, 1996. dermabrasão, transplante capilar ou transplante autólogo de gordura mostraram-se seguros, quando realizados em regiões não-inflamadas ${ }^{(73)}$.

\section{CONCLUSÃO}

As lesões cutâneas do lúpus eritematoso são as mais freqüentes manifestações da doença. Na maioria dos casos, as medidas de cuidados gerais, como proteção solar e uso de corticóides tópicos e de antimaláricos, consideradas terapias de primeira e segunda linhas, são efetivas no tratamento das lesões.

Entretanto, nos casos nos quais as lesões cutâneas sejam mais graves ou de difícil controle, o uso de drogas de terceira e quarta linhas, como retinóides e imunossupressores, faz-se necessário.

Mais recentemente, agentes biológicos, alguns amplamente utilizados no tratamento de doenças auto-imunes e outros ainda em investigação, parecem ter papel promissor na abordagem terapêutica dos casos refratários. No entanto, ainda são necessários mais estudos que comprovem a eficácia terapêutica dessas medicações no tratamento do lúpus cutâneo.

10. Wallace DJ: Principles of therapy and local measures. In: Wallace DJ, Hahn BH. Dubois' lupus erythematosus. 6. ed. Philadelphia: Lippincott Willians \& Wilkins; 2002. p. 1131-40.

11. Lin JH, Dutz JP, Sontheimer RD, Werth VP: Pathophysiology of cutaneous lupus erythematosus. Clin Rev Allergy Immunol 33(1-2): 85-106, 2007.

12. Bijl M, Kallenberg CG: Ultraviolet light and cutaneous lupus. Lupus 15(11): 724-7, 2006.

13. Bang B, Rygaard J, Baadsgaard O, Skov L: Increased expression of Fas on human epidermal cells after in vivo exposure to single-dose ultraviolet (UV) B or long-wave UVA radiation. $\mathrm{Br}$ J Dermatol 147(6): 1199-206, 2002.

14. Reefman E, Kuiper H, Jonkman MF, et al.: Skin sensitivity to UVB irradiation in systemic lupus erythematosus is not related to the level of apoptosis induction in keratinocytes. Rheumatology (Oxford) 45(5): 538-44, 2006.

15. Mattews JD, Whinttinggham S, Hopper BM, et al.: Association of auto antibodies with smoking, cardiovascular morbity and death in the Busselton population. Lancet 2: 754-8, 1973.

16. Regius O, Lengyl E, Borzsonyi L, et al.: The effect of smoking on the presence of antinuclear antibodies and on the morphology of lymphocytes in aged subjects. Z Gerontol 21: 161-3, 1988.

17. Brown K, Petri M, Goldman D: Cutaneous of SLE associated with other manifestations of SLE and with smoking (abstract). Arthitis Rheum 38: R27, 1995.

18. Lardet D, Martin S, Truchetet F, et al.: Effect of smokin g on the effectiveness of antimalarial drugs for cutaneous lesions of 
patients with lupus: assessment in a prospective study. Rev Med Interne 25(11): 786-91, 2004.

19. Rahman P, Gladman DD, Urowitz B: Smoking interferes with efficacy of antimalarical therapy in cutaneous lupus. J Rheumatol 25: 1716-9, 1998.

20. Jewell ML, McCauliffe DP: Patients with cutaneous lupus erythematosus who smoke are less responsive to antimalarial treatment. J Am Acad Dermatol 42 (6): 983-7, 2000.

21. Werth T: Current treatment of cutaneous lupus erythematosus. Dermat Online J 7 (1): 174-8, 2001.

22. Weston WL: Topical corticosteroids in dermatologic disorders. Hosp Pract 19: 159-72, 1984

23. Anonymous: Clobetasola potent new topical corticosteroid. Med Lett 28: 57-9, 1986.

24. Page F: Treatment of lupus erythematosus with mepacrine. Lancet 2: 755-8, 1951.

25. Wozniacka A, McCauliffe DP: Optimal use of antimalarials in treating cutaneous lupus erythematosus. Am J Clin Dermatol 6(1): 1-11, 2005.

26. Callen JP: Management of antimalarial refractory cutaneous lupus erythematosus. Lupus 6: 203-8, 1997.

27. Feldman R, Salmon D, Saurat JH: The association of the two antimalarials chloroquine and quinacrine for the treatmentresistant chronic and subacute cutaneous lupus erythematosus. Dermatology 189(4): 425-7, 1994.

28. Fabbri P, Cardinali C, Giomi B, Caproni M: Cutaneous lupus erythematosus: diagnosis and management. Am J Clin Dermatol 4 (7): 449-65, 2003.

29. Vena GA, Coviello C, Angelini G: Use of oral isotretinoin in the treatment of cutaneous lupus erythematosus. G Ital Dermatol Venereol 124 (6): 311-5, 1989.

30. Ruzicka T, Sommerburg C, Goerz G, et al.: Treatment of cutaneous lupus erythematosus with acitretin and hydroxychloroquine. $\mathrm{Br}$ J Dermatol 127: 513-8, 1992.

31. Sontheimer RD, McCauliffe DP: Cutaneous manifestations of lupus erythematosus. In: Wallace DJ, Hahn BH. Dubois' lupus erythematosus. 6. ed. Philadelphia: Lippincott Willians \& Wilkins; 2002. p. 573-618.

32. Jessop S, Whitelaw D, Jordan F: Drugs for discoid lupus erythematosus. Cochrane Database Syst Rev (1): CD 002954, 2001.

33. Sticherling M, Bonsmann G, Kuhn A: Diagnostic approach and treatment of cutaneous lupu s erythematosus. J Dtsch Dermatol Ges 6(1): 48-59, 2008 Jan.

34. Callen JP, Spencer LV, Burruss JB, Haltman J: Azathioprine. An effective, corticosteroid-sparing therapy for patients with recalcitrant cutaneous lupus erythematosus or with recalcitrant cutaneous leukocytoclastic vasculitis. Arch Dermatol 127 (4): 515-22, 1991.

35. Davis JC, Klippel JH: Antimalarials and immunossupressive therapies. In: Lahita RG, editor. Systemic lupus erythematosus. 4 ed. 2004. p. 1279-81.

36. Rothenberg, RJ, Graziano FM, Grandone JT, et al.: The use of methotrexate in steroid-resistant systemic lupus erythematosus. Arthritis Rheum 31: 612-5, 1998.

37. Walz LeBlanc BA, Dagenais P, Urowitz MB, et al.: Methotrexate in systemic lupus erythematosus. J Rheumatol 21: 836, 1994.
38. Carneiro JR, Sato EI: Double blind, randomized, placebo controlled clinical trial of methotrexate in systemic lupus erythematosus. J Rheumatol 26(6): 1275-9, 1999.

39. Wenzel J, Brähler S, Bauer R, et al.: Efficacy and safety of methotrexate. in recalcitrant cutaneous lupus erythematosus: results of a retrospective study in 43 patients. Br J Dermatol 153 (1): 157-62, 2005.

40. Remer CF, Weisman MH, Wallace DJ: Benefits of leflunomide in systemic lupus erythematosus: a pilot observational study. Lupus 10 (7): 480-3, 2001.

41. Suess A, Sticherling M: Leflunomide in subacute cutaneous lupus erythematosus - two sides of a coin. Int J Dermatol 47(1): 83-6, 2008.

42. Elias AR, Tam CC, David-Bajar KM: Subacute cutaneous lupus erythematosus associated with leflunomide. Cutis 76(3): 189-92, 2005.

43. Chan SK, Hazleman BL, Burrows NP: Subacute cutaneous lupus erythematosus precipitated by leflunomide. Clin Exp Dermatol 30(6): 724-5, 2005.

44. Goëb V, Berthelot JM, Joly P, et al.: Leflunomide-induced subacute cutaneous lupus erythematosus. Rheumatology (Oxford) 44(6): 823-4, 2005.

45. Holtman JH, Neustadt DH, Klein J, et al.: Dapsone is an effective therapy for the skin lesions of subacute cutaneous lupus erythematosus and urticarial vasculitis in a patient with C2 deficiency. J Rheumatol 17: 1222-5, 1990.

46. Cotell S, Robinson ND, Chan LS: Autoimmune blistering skin diseases. Am J Emerg Med 18: 288-99, 2000.

47. Ujiie H, Shimizu T, Ito M, et al.: Lupus erythematosus profundus successfully treated with dapsone: review of the literature. Arch Dermatol 142(3): 399-401, 2006.

48. Atra E, Sato EI: Treatment of the cutaneous lesions of systemic lupus erythematosus with thalidomide. Clin Exp Rheumatol 11(5): 487-93, 1993.

49. Coelho A, Souto MI, Cardoso CR, et al.: Long-term thalidomide use in refractory cutaneous lesions erythematosus: a 65 series of Brazilian patients. Lupus 14(60): 434-9, 2005.

50. Pisoni CN, Obermoser G, Cuadrado MJ, et al.: Skin manifestations of systemic lupus erythematosus refractory to multiple treatment modalities: poor results with mycophenolate mofetil. Clin Exp Rheumatol 23 (3): 393-6, 2005.

51. Schanz S, Ulmer A, Rassner G, Fierlbeck G: Successful treatment of subacute cutaneous lupus erythematosus with mycophenolate mofetil. Br J Dermatol 147 (1): 174-8, 2002.

52. Wenzel J, Bieber T, Uerlich M, Tüting T: Systemic treatment of cutaneous lupus erythematosus. J Dtsch Dermatol Ges 1(9): 694-704, 2003.

53. Luger T, Paul C: Potential new indications of topical calcineurin inhibitors. Dermatology 215(Suppl 1): 45-54, 2007.

54. Lampropoulos CE, Sangle S, Harrison P, et al.: Topical tacrolimus therapy of resistant cutaneous lesions in lupus erythematosus: a possible alternative. Rheumatology 43 (11): 1383-5, 2004.

55. Sugano M, Shintani Y, Kobayashi K, et al.: Successful treatment with topical tacrolimus in four cases of discoid lupus erythematosus. J Dermatol 33 (12): 887-91, 2006. 
56. Wollina U, Hansel G: The use of topical calcineurin inhibitors in lupus erythematosus: an overview J Eur Acad Dermatol Venereol 22(1): 1-6, 2008.

57. Tzellos TG, Kouvelas D: Topical tacrolimus and pimecrolimus in the treatment of cutaneous lupus erythematosus: an evidencebased evaluation. Eur J Clin Pharmacol 64(4): 337-41, 2008.

58. Yoshimasu T, Ohtani T, Sakamoto T, et al.: Topical FK506 (tacrolimus) therapy for facial erythematous lesions of cutaneous lupus erythematosus and dermatomyositis. Eur J Dermatol 12 (4): 387-8, 2002.

59. Callen JP: Treatment of cutaneous lesions in patients with lupus erythematosus. Dermatol Clin 12(1): 201-6, 1994.

60. Langlo L: Efficiency of local application of chloroquine and mepacrine in preventing the effects of ultraviolet rays in human skin. Acta Dermatolovener 37: 85-7, 1957.

61. Pelzig A, Witten VH, Sulzberger MB: Chloroquine for chronic discoid lupus erythematosus. Intralesional injections. Arch Dermatol 83: 146-8, 1961.

62. Zandman-Goddard G, Shoenfeld Y: Novel approaches to therapy for SLE. Clin Rev Allergy Immunol 25(1): 105-12, 2003.

63. Pelle MT: Issues and advances in the management and pathogenesis of cutaneous lupus erythematosus. Adv Dermatol 22: 55-65, 2006.

64. Rott $S$, Mrowietz U: The use of infliximab in dermatology. J Dtsch Dermatol Ges 5(8): 655-60, 2007.
65. Bano S, Bombardieri S, Doria A, et al.: Lupus erythematosus and the skin. Clin Exp Rheumatol 24(1 Suppl 40): S26-35, 2006.

66. Scheinfeld N: The medical uses and side effects of etanercept with a focus on cutaneous disease. J Drugs Dermatol 3(6): 653-9, 2004.

67. Thivolet J, Nicolas JF, Kanitakis J, et al.: Recombinant interferon $2 \mathrm{a}$ is effective in the treatment of discoid and subacute cutaneous lupus erythematosus. Br J Dermatol 122: 405-9, 1990.

68. Nicolas JF, Thivolet J, Kanitakis J, et al.: Response of discoid and subacute cutaneous lupus erythematosus.to recombinat interferon 2a. Br J Dermatol 95: 142S-5, 1990.

69. Wenzel J, Tüting T: Identification of type I interferonassociated inflammation in the pathogenesis of cutaneous lupus erythematosus opens up options for novel therapeutic approaches. Exp Dermatol 16(5): 454-63, 2007.

70. Lehmann P, Holzle E, Kind P, et al.: Experimental reprodution of skin lesions in lupus erythematosus by UV-A and UV-B radiation. J Am Acad Dermatol 22: 181-7, 1990.

71. Nived O, Johansen PB, Sturfelt G: Standartized ultraviolet-A exposure provokes skin reaction in systemis lupus erythematosus. Lupus 2: 247-50, 1993.

72. Dawe RS. Ultraviolet Al phototherapy. Br J Dermatol 148(4): 626-37, 2003.

73. Pinski KS, Roenigk Jr HH: Autologus fat tranplantation. Long term follow-up. J Dermatol Surg Oncol 18: 179-84, 1992. 